\title{
Controlling magnetic Feshbach resonances in polar open-shell molecules with non-resonant light
}

\author{
Michał Tomza, ${ }^{1,2,3}$ Rosario González-Férez, ${ }^{2,4}$ Christiane P. Koch, ${ }^{3, *}$ and Robert Moszynski ${ }^{1}$ \\ ${ }^{1}$ Faculty of Chemistry, University of Warsaw, Pasteura 1, 02-093 Warsaw, Poland \\ ${ }^{2}$ Instituto 'Carlos I' de Física Teórica y Computacional and Departamento de Física Atómica, \\ Molecular y Nuclear, Universidad de Granada, 18071 Granada, Spain \\ ${ }^{3}$ Theoretische Physik, Universität Kassel, Heinrich-Plett-Str. 40, 34132 Kassel, Germany \\ ${ }^{4}$ The Hamburg Center for Ultrafast Imaging, University of Hamburg, 22761 Hamburg, Germany
}

(Dated: October 29, 2018)

\begin{abstract}
Magnetically tunable Feshbach resonances for polar paramagnetic ground-state diatomics are too narrow to allow for magnetoassociation starting from trapped, ultracold atoms. We show that nonresonant light can be used to engineer the Feshbach resonances in their position and width. For non-resonant field intensities of the order of $10^{9} \mathrm{~W} / \mathrm{cm}^{2}$, we find the width to be increased by three orders of magnitude, reaching a few Gauss. This opens the way for producing ultracold molecules with sizeable electric and magnetic dipole moments and thus for many-body quantum simulations with such particles.
\end{abstract}

PACS numbers: 34.50.Cx,34.50.Rk,67.85.-d

Ultracold polar molecules are predicted to probe fundamental physics [1] and realize a wealth of many-body phenomena such as exotic quantum phases [2]. They are thus attracting significant interest in both the AMO and condensed matter communities [3]. Polar alkali dimers have already been produced in their absolute internal ground state close to quantum degeneracy [4], opening the way toward ultracold chemistry $[5,6]$ and quantum simulation [7]. Contrary to ground-state alkali dimers which are closed-shell, diatomics consisting of an open-shell and a closed-shell atom possess an unpaired electron, endowing the molecule with spin structure and a significant magnetic dipole moment. Since these molecules have both electric and magnetic dipoles, they are supreme candidates for creating topologically ordered states [8], investigating collective spin excitations [9] and realizing lattice-spin models [7]. While numerous ultracold mixtures of open-shell alkali and closedshell $\mathrm{Yb}$ or $\mathrm{Sr}$ atoms have already been studied experimentally [10-15], magnetoassociating the atoms into molecules has remained an elusive goal.

The most successful and widely used routes to producing ultracold dimers utilize magnetically tunable Feshbach resonances (FRs) [16, 17] where the hyperfine interaction couples a scattering state to a bound molecular level. Somewhat unexpectedly, FRs have been predicted for diatomics with a ${ }^{2} \Sigma$ ground electronic state such as $\mathrm{RbSr}$ and $\operatorname{LiYb}[18,19]$. The resonances are caused by a modification of the alkali atom's hyperfine structure due to the presence of the other atom [18] or by creating a hyperfine coupling due to the alkali atom polarizing the nuclear spin density of fermionic $\mathrm{Yb}$ [19]. However, the width of these resonances does not exceed a few milli-Gauss. This renders their use in magnetoassociation very difficult, if not impossible. A different kind of FR for a closed-shell/open-shell mixture has recently been observed, with one of the atoms in an electronically excited state $[20,21]$. In this case, the FR is induced by the anisotropy of the interaction between $S$-state and $P$-state atoms. Due to the finite excited state lifetime, such a FR is not ideally suited for making molecules. It suggests, however, to harness an anisotropic interaction for magnetoassociation.

Here we show that non-resonant light, which universally couples to the polarizability anisotropy of a molecule, induces FRs and modifies their position and width. This is due to the non-resonant light changing the background scattering length and altering the differential magnetic susceptibility. Our approach is related to dc electric field control of polar molecules [22-24] but comes with much more favorable requirements in terms of experimental feasibility. We find widths of a few Gauss for non-resonant field intensities of the order $10^{9} \mathrm{~W} / \mathrm{cm}^{2}$ for a wide range of polar open-shell molecular species. Widths of a few Gauss are sufficient for magnetoassociation. Non-resonant field control thus paves the way to producing ultracold particles with sizeable electric and magnetic dipole moment.

Magnetoassociation can employ an adiabatic ramp of the magnetic field across the resonance or a timedependent magnetic or radio-frequency (rf) field that drives a transition from a scattering state to a molecular level [17]. These two routes imply different requirements on the characteristics of the resonance. In both cases, a broad FR is needed. Adiabatic passage additionally requires a large product of width, $\Delta$, and background scattering length, $a_{b g}$. This is seen in the atom-molecule conversion efficiency, given by the Landau-Zener formula $1-\exp \left[-\eta n \frac{\hbar}{\mu}\left|\frac{a_{b g} \Delta}{B}\right|\right]$ with $n$ the atomic number density, $\dot{B}$ the magnetic field ramp speed, $\mu$ the reduced mass and $\eta$ a dimensionless prefactor [25]. Using Fermi's Golden 
Rule, the resonance width $\Delta$ can be estimated,

$$
\Delta \sim \frac{|\langle v|H| k\rangle|^{2}}{a_{b g} \delta \chi},
$$

in terms of the coupling $\langle v|H| k\rangle$ between molecular level $|v\rangle$ and scattering states $|k\rangle$, the background scattering length $a_{b g}$, and the differential magnetic susceptibility, $\delta \chi[26]$. The latter is simply the difference in slope of the bound and continuum energies as function of magnetic field at resonance. When the background scattering length $a_{b g}$ is larger than the mean scattering length $\bar{a}\left(\bar{a} \approx 0.48\left(2 \mu C_{6} / \hbar\right)^{1 / 4}\right.$ with $C_{6}$ the dispersion coefficient), the coupling $|\langle v|H| k\rangle|$ becomes proportional to $a_{b g}$. The width is then determined by background scattering length and differential magnetic susceptibility, $\Delta \sim a_{b g} / \delta \chi[26]$. The key point of our proposal is that both $\delta \chi$ and $a_{b g}$ can be tuned by applying a non-resonant field. This leads to significant changes in the resonance width $\Delta$ and the adiabaticity parameter $\left|a_{b g} \Delta\right|$.

The Hamiltonian describing the relative nuclear motion of an open-shell ${ }^{2} S$ atom, $a$, and a closed-shell ${ }^{1} S$ atom, $b$, reads

$$
\hat{H}=\frac{\hbar^{2}}{2 \mu}\left(-\frac{1}{r} \frac{d^{2}}{d r^{2}} r+\frac{\hat{L}^{2}}{r^{2}}\right)+\hat{H}_{a}+\hat{H}_{b}+V(r, \theta),
$$

where $r$ is the interatomic separation, $\hat{L}$ the rotational angular momentum operator, and $\theta$ the angle between the molecular axis and the space-fixed $Z$-axis. The atomic Hamiltonian including Zeeman and hyperfine interactions is given by

$$
\hat{H}_{j}=\zeta_{j} \hat{i}_{j} \cdot \hat{s}_{j}+\left(g_{e} \mu_{B} \hat{s}_{j, z}+g_{j} \mu_{N} \hat{i}_{j, z}\right) B
$$

with $\hat{s}_{j}$ and $\hat{i}_{j}$ the electron and nuclear spin operators, $g_{e / j}$ the electron and nuclear $g$ factors, and $\mu_{B / N}$ the Bohr and nuclear magnetons. $\zeta_{j}$ denotes the hyperfine coupling constant. For a fermionic closed-shell ${ }^{1} S$ atom, Eq. (3) reduces to the nuclear Zeeman term, whereas for a bosonic one it is zero. The interatomic interaction reads

$$
\begin{aligned}
V(r, \theta)= & V_{X^{2} \Sigma^{+}}(r)+\Delta \zeta_{a}(r) \hat{i}_{a} \cdot \hat{s}_{a} \\
& -\frac{I}{2 \epsilon_{0} c}\left(\alpha_{\perp}(r)+\Delta \alpha(r) \cos ^{2} \theta\right)
\end{aligned}
$$

for magnetic and non-resonant laser fields parallel to the space-fixed $Z$-axis. $V_{X^{2} \Sigma^{+}}(r)$ is the potential energy curve for the $X^{2} \Sigma^{+}$ground electronic state, and $\Delta \zeta_{a}(r)$ the interaction-induced variation of the hyperfine coupling $[18,19]$. The molecular static polarizability with perpendicular component $\alpha_{\perp}(r)$ and anisotropy $\Delta \alpha(r)$ couples to non-resonant light of intensity $I$, linearly polarized along the space-fixed $Z$-axis. We omit spin-rotation couplings as well as the coupling resulting from a non-zero nuclear spin of a fermionic closed-shell atom since they are significantly smaller than $\Delta \zeta_{a}(r)$.

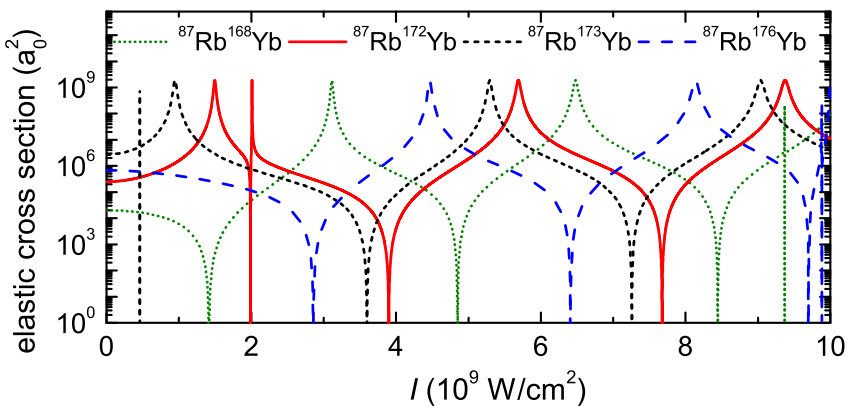

FIG. 1: (Color online) Non-resonant light control of scattering properties: Elastic cross section as a function of the nonresonant light intensity $\left(E / k_{B}=100 \mathrm{nK}, B=0\right)$.

We focus on RbYb for which spectroscopic and $a b$ initio data for the interaction potential are available [27]. The $r$-dependent isotropic and anisotropic polarizabilities are calculated using state of the art coupled cluster methods, small-core energy consistent pseudopotentials, and large basis sets [28]. They perfectly agree with Silberstein's formula $[29,30]$ evaluated for the atomic polarizabilities of Ref. [31]. The interaction-induced variation of the hyperfine coupling, $\Delta \zeta_{a}(r)$, is taken from Ref. [26]. The total scattering wave function is constructed in an uncoupled basis set, $\left|i_{a}, m_{i, a}\right\rangle\left|s_{a}, m_{s, a}\right\rangle\left|L, m_{L}\right\rangle$ with $m_{j}$ the projection of angular momentum $j$ on the space-fixed $Z$ axis, assuming the projection of the total angular momentum of rubidium $m_{f}=m_{i, a}+m_{s, a}$ to be conserved. The coupled channels equations are solved using a renormalized Numerov propagator [32]. The scattering lengths and elastic cross sections are obtained from the $S$ matrix for the entrance channel, $a=\left(1-S_{11}\right) /\left(1+S_{11}\right) /(i k)$ and $\sigma_{e l}=\pi\left|1-S_{11}\right| / k^{2}$, with $k=\sqrt{2 \mu E} / \hbar$ and $E$ the collision energy, assumed to be $100 \mathrm{nK}$. The resonance width $\Delta$ is determined by fitting the scattering length to $a(B)=a_{b g}\left(1-\Delta /\left(B-B_{r e s}\right)\right)[16,17]$.

Non-resonant light modifies the energies of rovibrational levels and scattering states alike [33-36]. The latter implies control of scattering properties such as the cross sections. This is illustrated by Fig. 1 which displays a series of maxima and minima of the elastic cross section as a function of non-resonant field intensity. The maxima correspond to a large absolute value of $a_{b g}$ and occur when a scattering state becomes bound; the minima indicate non-interacting atoms. Broad maxima of the elastic cross section are observed when an $s$-wave scattering state is pushed below threshold, whereas the narrow features in Fig. 1 are caused by higher partial waves. New FRs are created by the non-resonant light shifting bound levels. This happens when a bound level crosses the atomic threshold of a different hyperfine level as indicated by the dots in Fig. 2a). New resonances, higher than $s$-wave, may also be induced by mixing partial waves or by spin-rotation coupling between higher 


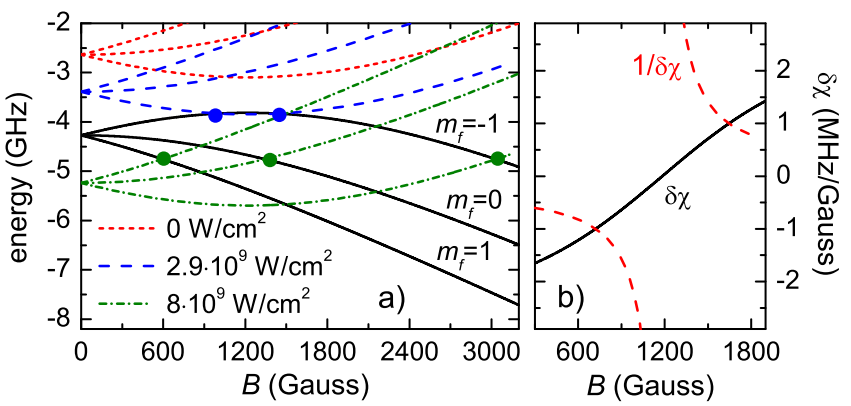

FIG. 2: (Color online) Creating new FR with non-resonant light: a) Atomic thresholds (solid black lines) start to cross molecular levels (dashed lines) as the non-resonant light shifts the level positions $\left({ }^{87} \mathrm{Rb}^{176} \mathrm{Yb}\right.$ with $\left.\left|m_{f}\right| \leq i_{a}-1 / 2\right)$. The dots indicate the position of the new FR. b) The level shifts are accompanied by a variation of the differential magnetic susceptibility $\delta \chi$ vs magnetic field $\left(m_{f}=-1, I=0\right)$.

partial waves. The non-resonant field dependence of the background scattering length observed in Fig. 1 and the creation of new FR due to the non-resonant light shown in Fig. 2 together with Eq. (1) suggest three mechanisms to increase the width of FRs: (i) $\delta \chi \rightarrow 0$, (ii) $\left|a_{b g}\right| \rightarrow \infty$, and (iii) $\left|a_{b g}\right| \rightarrow 0$. In case (i), $\left|a_{b g} \Delta\right|$ becomes large unless it coincides with case (iii), and large $\left|a_{b g} \Delta\right|$ is guaranteed in case (ii). Then both adiabatic ramping across the resonance and rf association are possible. In contrast, $\left|a_{b g} \Delta\right|$ will always stay small in case (iii), preventing adiabatic passage. Since adiabatic ramping is the most popular technique for magnetoassociation, we focus on cases (i) and (ii) here and will report on case (iii) elsewhere [28].

We find that case (i) yields the largest widths. This is illustrated in Fig. 3a) for ${ }^{87} \mathrm{Rb}^{176} \mathrm{Yb}$ : A pair of resonances is created when the molecular level crosses an atomic threshold close to the maximum of its magnetic field dependence, cf. blue dots in Fig. 2a). The resonances come with a very large width $\Delta$, of the order of a few Gauss, cf. the left peak in Fig. 3a), and are separated by several Gauss (by $6 \mathrm{G}$ for example for $\Delta \approx 3 \mathrm{G}$ ). The large width is rationalized by the broad pole of $1 / \delta \chi$ shown in Fig. 2b) which enters Eq. (1). Not only the width $\Delta$ but also the adiabaticity parameter $\left|a_{b g} \Delta\right|$ is found to be large, of the order of $10 \mathrm{a}_{0} \cdot \mathrm{G}$, whereas the background scattering length remains comparatively small, of the order of $10 \mathrm{a}_{0}$. A second peak of the width $\Delta$, of the order of $10 \mathrm{G}$, is observed in Fig. 3a), at $I=2.88 \cdot 10^{9} \mathrm{~W} / \mathrm{cm}^{2}$. It is caused by $a_{b g}$ going to zero, which can be inferred from the corresponding minimum of the blue dashed curve in Fig. 1. The joint occurrence of $\delta \chi \rightarrow 0$ and $a_{b g} \rightarrow 0$ is a coincidence. As can be seen in Fig. 3a) and b), such a coincidence leads to particularly broad resonances for a range of non-resonant field intensities which at the same time are separated by

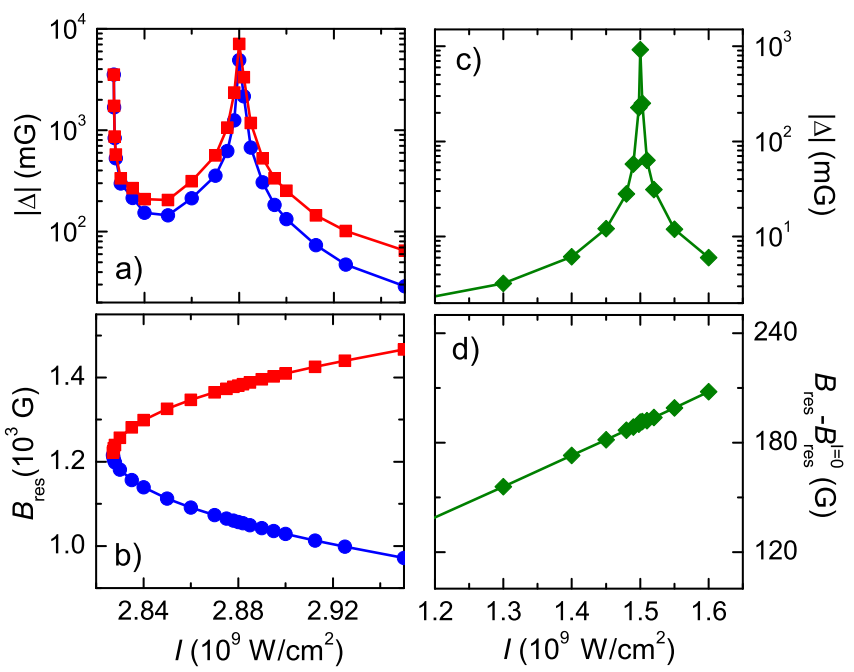

FIG. 3: (Color online) Controlling the width of a FR by modifying $\delta \chi(\mathrm{a}, \mathrm{b})$ : Resonance width $\Delta$ and resonance position $B_{\text {res }}$ vs non-resonant light intensity for ${ }^{87} \mathrm{Rb}^{176} \mathrm{Yb}$ and the pair of resonances indicated by blue dots in Fig. $2\left(m_{f}=-1\right.$, $\left.B_{\text {res }}=1219 \mathrm{G}\right)$. Controlling the width of a FR by tuning $a_{b g}$ to large values $(\mathrm{c}, \mathrm{d})$ : Resonance width $\Delta$ and change in resonance position $B_{\text {res }}-B_{\text {res }}^{I=0}$ vs non-resonant light intensity for ${ }^{87} \mathrm{Rb}^{172} \mathrm{Yb}\left(m_{f}=1, B_{\text {res }}^{I=0}=1592 \mathrm{G}\right)$.

several hundreds Gauss. However, due to $a_{b g} \rightarrow 0$, the adiabaticity parameter $\left|a_{b g} \Delta\right|$ remains small. The adiabaticity parameter is guaranteed to be large in case (ii) when the non-resonant field is used to tune the background scattering length to very large values. This is illustrated in Fig. 3c) and d). The maximum width $\Delta$ which is not limited in theory will depend on the stability of the non-resonant field intensity in practice. For example, an increase by $10^{3}$ requires intensity stabiliziation of the order $10^{-3}$ to $10^{-4}$. The actual value of $\Delta$ that can be obtained also depends on the field-free width. But even for very narrow resonances, with the field-free $\Delta$ below $1 \mathrm{mG}$, the engineered width easily reaches $100 \mathrm{mG}$, as demonstrated by Fig. 3c).

We find non-resonant light intensities of the order of $10^{9} \mathrm{~W} / \mathrm{cm}^{2}$ to be sufficient to create FRs for all isotopologues of RbYb. The smallest intensity is required for diatomics with a molecular level just above the atomic threshold since the non-resonant field always lowers the energy in the electronic ground state [36]. For example, a pair of broad resonances as shown in Fig. 3a,b) is also observed for ${ }^{85} \mathrm{Rb}^{170} \mathrm{Yb}$ (with $\Delta>0.5 \mathrm{G}$ at $I=1.29 \cdot 10^{9} \mathrm{~W} / \mathrm{cm}^{2}$ ). When only the rubidium isotope is exchanged, the dependence on the non-resonant light intensity remains essentially unchanged compared to Fig. 3a,b). Of course, different hyperfine levels may come into play, e.g., $m_{f}=-2$ or $m_{f}=-1$, which imply different magnetic fields $\left(B_{\text {res }}=722 \mathrm{G}\right.$ and $B_{\text {res }}=$ $361 \mathrm{G}$, respectively, for $\left.{ }^{85} \mathrm{Rb}^{176} \mathrm{Yb}\right)$. The left peak of 
$\Delta$ in Fig. 3a) and the associated increase in $\left|a_{b g} \Delta\right|$ is found for all RbYb isotopologues. The right peak corresponds to a coincidence of case (i) with case (iii) and is specific to ${ }^{87} \mathrm{Rb}^{176} \mathrm{Yb}$. Case (i) may coincide also with case (ii). This happens for ${ }^{87} \mathrm{Rb}^{174} \mathrm{Yb}$, yielding an adiabaticity parameter $\left|a_{b g} \Delta\right|$ of the order of $100 \mathrm{a}_{0}$. G. Case (ii), i.e., large $a_{b g}$, is most easily realized for molecules with a large and negative field-free background scattering length $a_{s}$. For ${ }^{87} \mathrm{Rb}^{172} \mathrm{Yb}$ shown in Fig. 3c,d) for example $a_{s}=-131 a_{0}$ [37]. Another good candidate for case (ii) is ${ }^{87} \mathrm{Rb}^{173} \mathrm{Yb}$ (with $a_{s}=-431 a_{0}[37]$ ).

The three mechanisms are generally applicable due to the universal coupling to non-resonant light. Notably, we find the characteristics of controlling the resonance width by tuning the background scattering length as shown in Fig. 3c,d) to be common to all ${ }^{2} \Sigma$ molecules. When considering closed-shell/open-shell mixtures other than $\mathrm{RbYb}$, different strengths of both magnetic field and nonresonant light might, however, be required. For example, LiYb has a smaller reduced mass than RbYb and Li a smaller polarizability than $\mathrm{Rb}$ which implies a larger non-resonant field intensity. The magnetic field strength for which a molecular level crosses the atomic threshold close to the maximum of its magnetic field dependence, relevant for case (i), is determined by the hyperfine splitting [26]. It is thus smaller for mixtures involving $\mathrm{Li}$, $\mathrm{Na}$ or $\mathrm{K}$ and larger for those involving Cs instead of Rb. Prospects are best for RbSr and $\mathrm{CsYb}[28]$ for which the interaction induced variation of the hyperfine structure and the polarizabilities are largest. Together with the tunability of the field-free background scattering length by choice of the $\mathrm{Yb}$ isotope, this makes $\mathrm{CsYb}$ in particular another very promising candidate.

When tuning non-resonant light and magnetic field for interspecies magnetoassociation, undesired losses may occur due to accidentally hitting an intraspecies FR or shape resonance. For example, for RbYb, depending on the isotope, one to three shape resonances are observed for $\mathrm{Yb}_{2}$ at non-resonant field intensities of the order of $10^{9} \mathrm{~W} / \mathrm{cm}^{2}$. The shape resonance found for ${ }^{176} \mathrm{Yb}_{2}$ at $I=3.05 \cdot 10^{9} \mathrm{~W} / \mathrm{cm}^{2}$ is sufficiently far from $I=$ $2.83 \cdot 10^{9} \mathrm{~W} / \mathrm{cm}^{2}$, for which the width of the ${ }^{87} \mathrm{Rb}^{176} \mathrm{Yb}$ FR is increased to several Gauss, cf. Fig. 3a). The separation is even slightly larger for ${ }^{174} \mathrm{Yb}_{2}$. Alkali intraspecies FRs are found to be shifted in position by the non-resonant field. If, as the result, an intraspecies FR is moved too close to the interspecies one, a different $\mathrm{Yb}$ isotope should be selected. Similarly, selection of the hyperfine level provides a solution, if a shape resonance approaches the interspecies FR too closely, for example for $\mathrm{CsYb}$. Perturbations due to intraspecies resonances can thus be avoided. Such losses do not occur altogether when working in a double-species Mott insulator state [38].

Our proposal for non-resonant light controlled magnetoassociation requires intensities of the order of
$10^{9} \mathrm{~W} / \mathrm{cm}^{2}$ and magnetic fields of the order of $1000 \mathrm{G}$. These requirements are within current experimental capabilities. Intensities of the order $10^{9} \mathrm{~W} / \mathrm{cm}^{2}$ can be achieved using intracavity beams with spot sizes of about $10 \mu \mathrm{m}$ and powers of the order of $1 \mathrm{~kW}$. Such spot sizes could be desirable for creating an additional trap. Larger spot sizes, up to $100 \mu \mathrm{m}$, are possible when using an optical buildup cavity [39, 40]. The required intensity can be stabilized at a level of 0.001 , but even $10^{-4}$ should be reachable with refined feedback techniques. Magnetic fields can be stabilized at the level $10^{-5}-10^{-6}$ [41] such that magnetic field stability is not a concern for the resonance widths and separations discussed here. Losses due to photon scattering can be kept minimal by choosing light, such as that of a $\mathrm{CO}_{2}$ laser, that is far off resonance with any molecular transition. Estimating the heating rates for $I=10^{9} \mathrm{~W} / \mathrm{cm}^{2}$ in terms of the atomic photon scattering rates [42], we find the largest heating rate, that of the alkali atom, to be only of the order of $1 \mathrm{nK} / \mathrm{s}$ for a wavelength of $10 \mu \mathrm{m}$. Wavelengths in the near infrared, e.g., $1064 \mathrm{~nm}$ or $1550 \mathrm{~nm}$, could also be employed. For the telecom wavelength, we find a heating rate of the order of $10 \mu \mathrm{K} / \mathrm{s}$. This should be sufficiently low to allow for adiabatic ramps whereas for $1064 \mathrm{~nm}$ with heating rates below $1 \mathrm{mK} / \mathrm{s}$, the experiment needs to be conducted within $1 \mathrm{~ms}$, better adapted to $\mathrm{rf}$ magnetoassociation [17]. The actually required intensities and associated heating rates for these wavelengths might, however, be lower due to the dynamic instead of the static polarizabilities coming into play. This will be studied in detail elsewhere [28].

Compared to electric field control of FRs for polar molecules [22-24], our proposal corresponds to more favorable experimental conditions. For diatomics consisting of an alkali atom and $\mathrm{Sr}$ or $\mathrm{Yb}$, we find electric fields of several hundreds $\mathrm{kV} / \mathrm{cm}$ to be required. This clearly exceeds current experimental capabilities. Compared to the permanent electric dipole moment coupling to a dc electric field, the interaction of Eq. (4) contains diagonal in addition to off-diagonal matrix elements in the basis of field-free rotational eigenstates. This explains the large shifts in level positions which allow in particular for mechanism (i), i.e., $\delta \chi \rightarrow 0$. Moreover, the permanent dipole moment vanishes as $1 / r^{7}$ compared to the asymptotic $1 / r^{3}$ behavior of the polarizability. These facts together explain the much better prospects in terms of experimental feasibility of our approach.

In conclusion, we have shown that non-resonant light can be used to control FRs of mixtures of openshell/closed-shell atoms, engineering their widths to reach up to a few Gauss. Such resonances are sufficiently broad for magnetoassociation. The required field strengths and control are all within current experimental capabilities. Our proposal opens the way for producing ultracold molecules with sizeable electric and magnetic dipole moments and thus for many-body quantum simulations with such particles. 
We would like to thank Piotr Żuchowski and Axel Görlitz for making their $\mathrm{RbYb}$ potentials available to us prior to publication and Michael Drewsen and Jun Ye for providing their expertise on the state of the art of laser technology. The authors enjoyed hospitality of the Kavli Institute of Theoretical Physics where the work reported here was initiated. MT is supported by the Foundation for Polish Science MPD Programme co-financed by the EU European Regional Development Fund. RGF acknowledges a Mildred Dresselhaus award from the excellence cluster 'The Hamburg Center for Ultrafast Imaging - Structure, Dynamics and Control of Matter at the Atomic Scale' of the Deutsche Forschungsgemeinschaft. Financial support by the Spanish project FIS2011-24540 (MICINN), the Grants P11-FQM-7276 and FQM-4643 (Junta de Andalucía), and Andalusian research group FQM-207, the project N-N204-215539 of the Polish Ministry of Science and Higher Education, the MISTRZ program of the Foundation for Polish Science, and in part by the National Science Foundation under Grant No. NSF PHY11-25915 is gratefully acknowledged.

* Electronic address: christiane.koch@uni-kassel.de

[1] D. DeMille, S. B. Cahn, D. Murphree, D. A. Rahmlow, and M. G. Kozlov, Phys. Rev. Lett. 100, 023003 (2008).

[2] N. Y. Yao, A. V. Gorshkov, C. R. Laumann, A. M. Läuchli, J. Ye, and M. D. Lukin, Phys. Rev. Lett. 110, 185302 (2013).

[3] L. D. Carr, D. DeMille, R. V. Krems, and J. Ye, New J. Phys. 11, 055049 (2009).

[4] K. K. Ni, S. Ospelkaus, M. H. G. de Miranda, A. Pe'er, B. Neyenhuis, J. J. Zirbel, S. Kotochigova, P. S. Julienne, D. S. Jin, and J. Ye, Science 322, 231 (2008).

[5] S. Ospelkaus, K.-K. Ni, D. Wang, M. H. G. de Miranda, B. Neyenhuis, G. Quéméner, P. S. Julienne, J. L. Bohn, D. S. Jin, and J. Ye, Science 327, 853 (2010).

[6] M. H. G. de Miranda, A. Chotia, B. Neyenhuis, D. Wang, G. Quéméner, S. Ospelkaus, J. L. Bohn, J. Ye, and D. S. Jin, Nat. Phys. 7, 502 (2011).

[7] A. Micheli, G. K. Brennen, and P. Zoller, Nat. Phys. 2, 341 (2006).

[8] I. Bloch, J. Dalibard, and W. Zwerger, Rev. Mod. Phys. 80, 885 (2008).

[9] J. Perez-Rios, F. Herrera, and R. V. Krems, New. J. Phys. 12 (2010).

[10] N. Nemitz, F. Baumer, F. Münchow, S. Tassy, and A. Görlitz, Phys. Rev. A 79, 061403 (2009).

[11] F. Baumer, F. Münchow, A. Görlitz, S. E. Maxwell, P. S. Julienne, and E. Tiesinga, Phys. Rev. A 83, 040702 (2011).

[12] H. Hara, Y. Takasu, Y. Yamaoka, J. M. Doyle, and Y. Takahashi, Phys. Rev. Lett. 106, 205304 (2011).

[13] V. V. Ivanov, A. Khramov, A. H. Hansen, W. H. Dowd, F. Münchow, A. O. Jamison, and S. Gupta, Phys. Rev. Lett. 106, 153201 (2011).

[14] B. Pasquiou, A. Bayerle, S. M. Tzanova, S. Stellmer,
J. Szczepkowski, M. Parigger, R. Grimm, and F. Schreck, Phys. Rev. A 88, 023601 (2013).

[15] T. Aoki, Y. Yamanaka, M. Takeuchi, Y. Torii, and Y. Sakemi, Phys. Rev. A 87, 063426 (2013).

[16] T. Köhler, K. Góral, and P. S. Julienne, Rev. Mod. Phys. 78, 1311 (2006).

[17] C. Chin, R. Grimm, P. S. Julienne, and E. Tiesinga, Rev. Mod. Phys. 82, 1225 (2010).

[18] P. S. Żuchowski, J. Aldegunde, and J. M. Hutson, Phys. Rev. Lett. 105, 153201 (2010).

[19] D. A. Brue and J. M. Hutson, Phys. Rev. Lett. 108, 043201 (2012).

[20] S. Kato, S. Sugawa, K. Shibata, R. Yamamoto, and Y. Takahashi, Phys. Rev. Lett. 110, 173201 (2013).

[21] A. Khramov, A. Hansen, W. Dowd, R. J. Roy, C. Makrides, A. Petrov, S. Kotochigova, and S. Gupta, Phys. Rev. Lett. 112, 033201 (2014).

[22] R. V. Krems, Phys. Rev. Lett. 96, 123202 (2006).

[23] Z. Li and R. V. Krems, Phys. Rev. A 75, 032709 (2007).

[24] B. Marcelis, B. Verhaar, and S. Kokkelmans, Phys. Rev. Lett. 100, 153201 (2008).

[25] E. Hodby, S. T. Thompson, C. A. Regal, M. Greiner, A. C. Wilson, D. S. Jin, E. A. Cornell, and C. E. Wieman, Phys. Rev. Lett. 94, 120402 (2005).

[26] D. A. Brue and J. M. Hutson, Phys. Rev. A 87, 052709 (2013).

[27] M. Borkowski, P. S. Żuchowski, R. Ciuryło, P. S. Julienne, D. Kędziera, L. Mentel, P. Tecmer, F. Münchow, C. Bruni, and A. Görlitz, Phys. Rev. A 88, 052708 (2013).

[28] M. Tomza, R. González-Férez, C. P. Koch, and R. Moszynski, in preparation.

[29] T. G. Heijmen, R. Moszyński, P. E. Wormer, and A. van der Avoird, Mol. Phys. 89, 81 (1996).

[30] L. Jensen, P.-O. Åstrand, A. Osted, J. Kongsted, and K. V. Mikkelsen, J. Chem. Phys. 116, 4001 (2002).

[31] A. Derevianko, S. G. Porsev, and J. F. Babb, At. Data Nucl. Data Tables 96, 323 (2010).

[32] B. R. Johnson, J. Chem. Phys. 69, 4678 (1978).

[33] M. Lemeshko and B. Friedrich, Phys. Rev. Lett. 103, 053003 (2009).

[34] M. Lemeshko and B. Friedrich, J. Phys. Chem. A 114, 9848 (2010).

[35] R. González-Férez and C. P. Koch, Phys. Rev. A 86, 063420 (2012).

[36] M. Tomza, W. Skomorowski, M. Musiał, R. GonzálezFérez, C. P. Koch, and R. Moszynski, Mol. Phys. 111, 1781 (2013).

[37] F. Münchow, Ph.D. thesis, Universität Düsseldorf (2012).

[38] H. Hara, H. Konishi, S. Nakajima, Y. Takasu, and Y. Takahashi, J. Phys. Soc. Jpn. 83, 014003 (2014).

[39] D. C. Yost, A. Cingöz, T. K. Allison, A. Ruehl, M. E. Fermann, I. Hartl, and J. Ye, Opt. Express 19, 23483 (2011).

[40] A. Cingöz, D. C. Yost, T. K. Allison, A. Ruehl, M. E. Fermann, I. Hart, and J. Ye, Nature 482, 68 (2012).

[41] G. Zürn, T. Lompe, A. N. Wenz, S. Jochim, P. S. Julienne, and J. M. Hutson, Phys. Rev. Lett. 110, 135301 (2013).

[42] R. Grimm, M. Weidemüller, and Y. B. Ovchinnikov, Adv. At. Mol. Opt. Phys. 42, 95 (2000). 